\title{
A comparison of parameters of 3-minute and 5-minute oscillations in sunspots from synchronous microwave and optical observations
}

\author{
V. E. Abramov-Maximov ${ }^{1}$, G. B. Gelfreikh ${ }^{1}$, N. I. Kobanov ${ }^{2}$ and \\ K. Shibasaki ${ }^{3}$ \\ ${ }^{1}$ Central astronomical observatory at Pulkovo, Russian Acad. Sci., \\ Pulkovskoe chaussee., 65/1, St. Petersburg, 196140, Russia \\ email: beam@gao.spb.ru \\ ${ }^{2}$ Institute of Solar-terrestrial Physics RAS SB, Lermontov St., 134, Irkutsk, 664033, Russia \\ ${ }^{3}$ Nobeyama Solar Radio Observatory, Minamimaki, Minamisaku, Nagano 384-1305, Japan
}

\begin{abstract}
The observations of 3 and 5 minute oscillations in sunspots present information on propagation of MHD waves in the magnetic tubes of sunspots. We present a comparison of wavelet spectra of radio flux oscillations at $\lambda=1.76 \mathrm{~cm}$ and oscillations of longitudinal component of the velocity at the chromosphere in sunspot umbra and penumbra in AR 10661 (2004, Aug 18). The radio maps of the Sun obtained with the Nobeyama Radioheliograph were used. The spatial resolution of the radio data was about 10-15 arcsec, and 10 sec cadence was used. On the radio maps sunspot-associated sources were identified and time profiles of their maximum brightness temperatures for each radio source were calculated. Radio data consists of information of oscillations of plasma parameters (in the regions with magnetic field $B=2000 \mathrm{G}$ ) at the level of the chromosphere-corona transition region. The optical observations were carried out at Sayan observatory. These data included information on longitude component of the magnetic field at the photosphere (line Fe I $6569 \AA$ ) and longitudinal component of the velocity at the chromosphere (line $H \alpha$ was used). Comparing the wavelet diagrams covering the same periods of observations at radio and optics showed that some wave trains of time profiles are very similar in both kinds of observations (similar oscillation frequencies and their drifts, variations of amplitudes), however, some significant differences were also registered. The best similarity in optical and radio oscillations was found when the active region (AR) was near the center of the solar disk. The phase shifts between the two kinds of observations reflecting the propagation of MHD waves were also analyzed.
\end{abstract}

Keywords. Sun: radio radiation, Sun: oscillations, waves, MHD

\section{Introduction}

The quasi-periodic oscillations are registered practically in all wavelength ranges and in all structures of the solar atmosphere (Bogdan 2000, Fludra 2001, Gelfreikh, Nagovitsyn \& Nagovitsyna 2006). Oscillations have periods from seconds to hours or even days. The dominant periods of oscillations for sunspots are 3 minutes and 5 minutes. Most of these oscillations are of unstable nature: both amplitude and frequency are varied with time, often seen as trains of about a dozen of periods. Studing of oscillations can help us to understand such fundamental astrophysical problems as accumulation and release of energy, physics of corona heating and flares origin. 


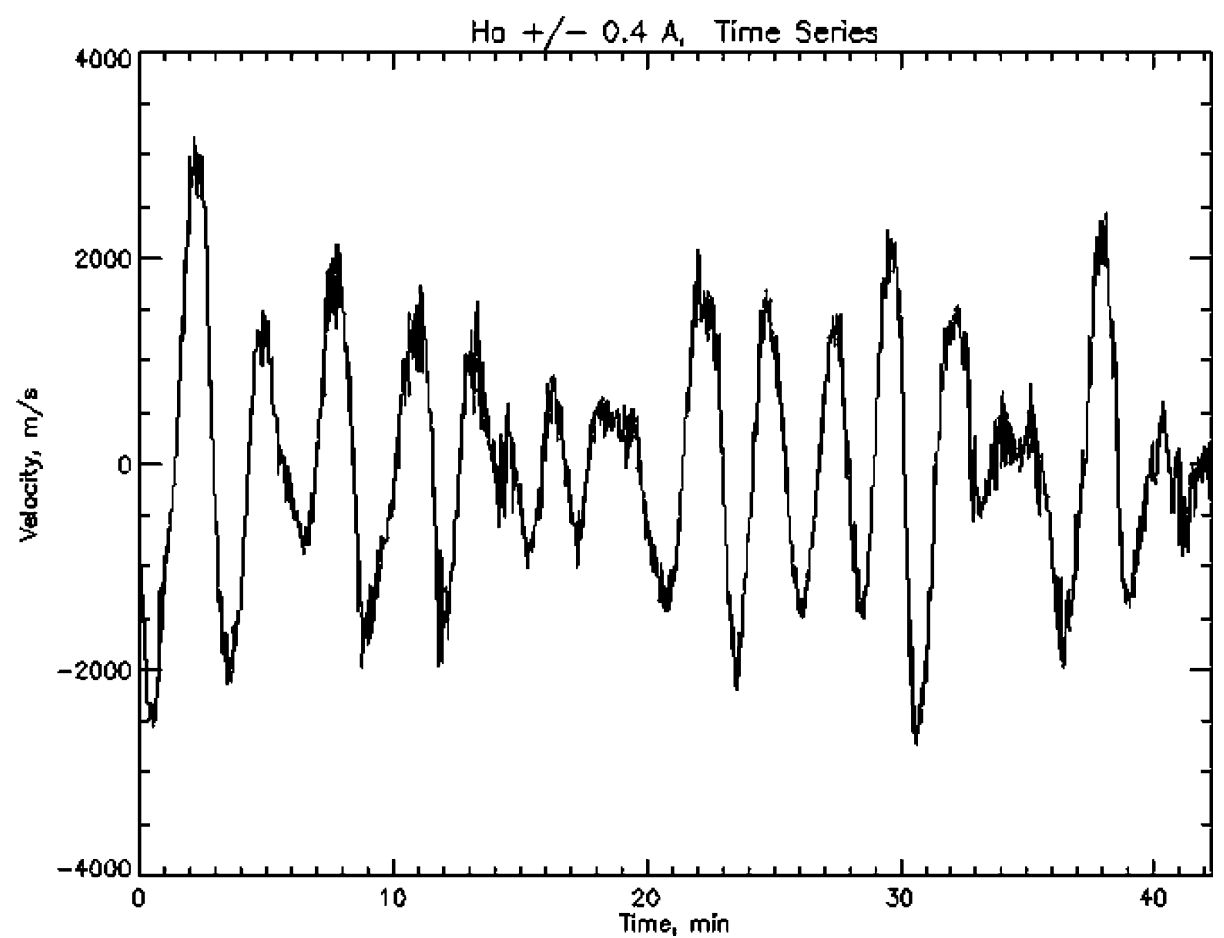

Figure 1. Time-variations of longitudinal component of the chromospheric velocity $(H \alpha$ observations), August 18, 2004, 01:01 - 01:43 UT, AR10661.

The choice of an adequate theory for interpretation of observations includes to essentially complicated problems. For analysis of the three-dimensional structure of the quasiperiodic oscillations it is necessary to use simultaneous observations in a few wavelength ranges. For many years the observations were limited by optical methods representing the phenomena at the level of the photosphere and chromosphere. Modern space techniques made a progress in the study of oscillations at the level of corona and transition region. However, the magnetic tubes of sunspots are not seen on such images like as it is seen from photosphere level. A new progress in such studies was achieved as a result of mapping of the Sun at microwaves. On such maps the sunspots are clearly seen as bright highly polarized small radio sources generated by thermal cyclotron emission of coronal electrons at lower harmonics of its gyro frequency; magnetic field of thousands of Gauss is needed for the effect.

\section{Observations}

Optical observations were carried out on horizontal solar telescope of the Sayan solar observatory of the Institute of Solar-Terrestrial Physics of Siberian Devision of RAS. The observatory is located at the height of $2000 \mathrm{~m}$ above sea level. Variations of the line-ofsight velocity and longitudinal component of the magnetic field (using line FeI $6559 \AA$ ) at the photosphere and the longitude velocity component in the chromospheric $H \alpha$ line. The slit of the spectrograph was East-West directed crossing the center of sunspot. The realized space resolution was about one arc sec along the spectrograph slit. The duration of one set of observations was about one hour with cadence of a few seconds. While analyzing the observations we used wavelet spectra with Morle functions of the 6-th order as a base function (Kobanov, Kolobov \& Makarchik 2006). 


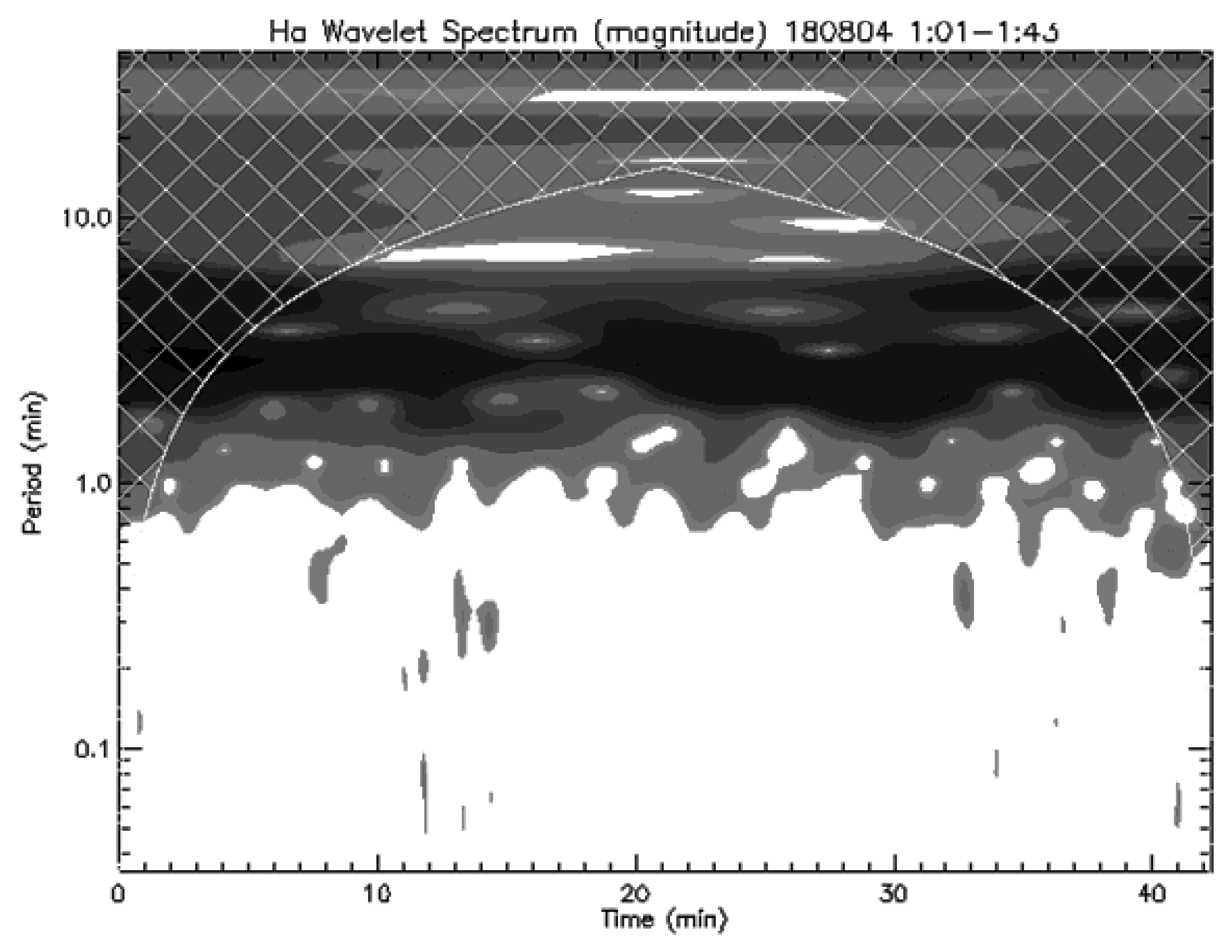

Figure 2. Wavelet spectrum of time-variations of longitudinal component of the chromospheric velocity ( $H \alpha$ observations), August 18, 2004, 01:01 - 01:43 UT, AR10661.

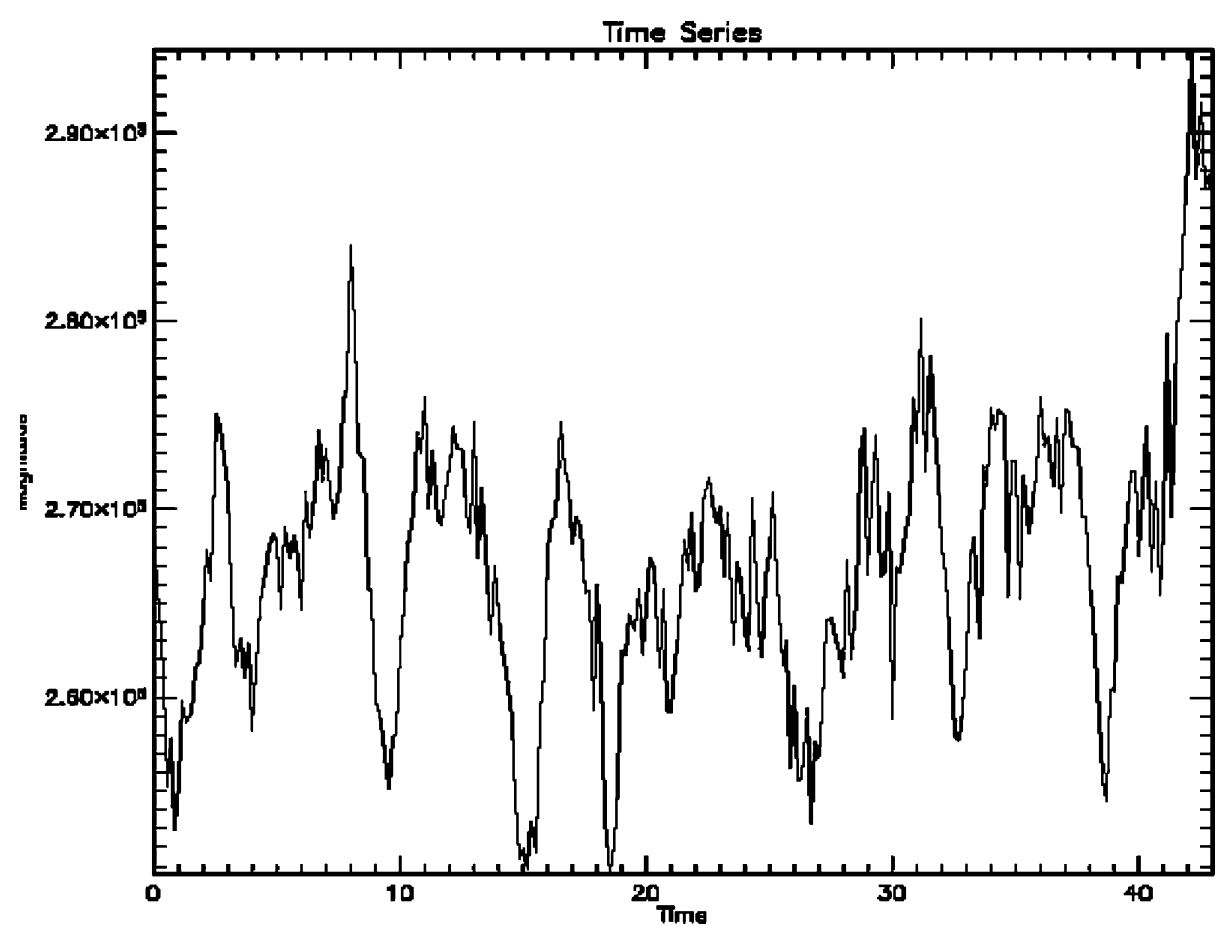

Figure 3. Variations of radio emission of the bright source in the active region AR10661, August 18, 2004, 01:01 - 01:43 UT. 


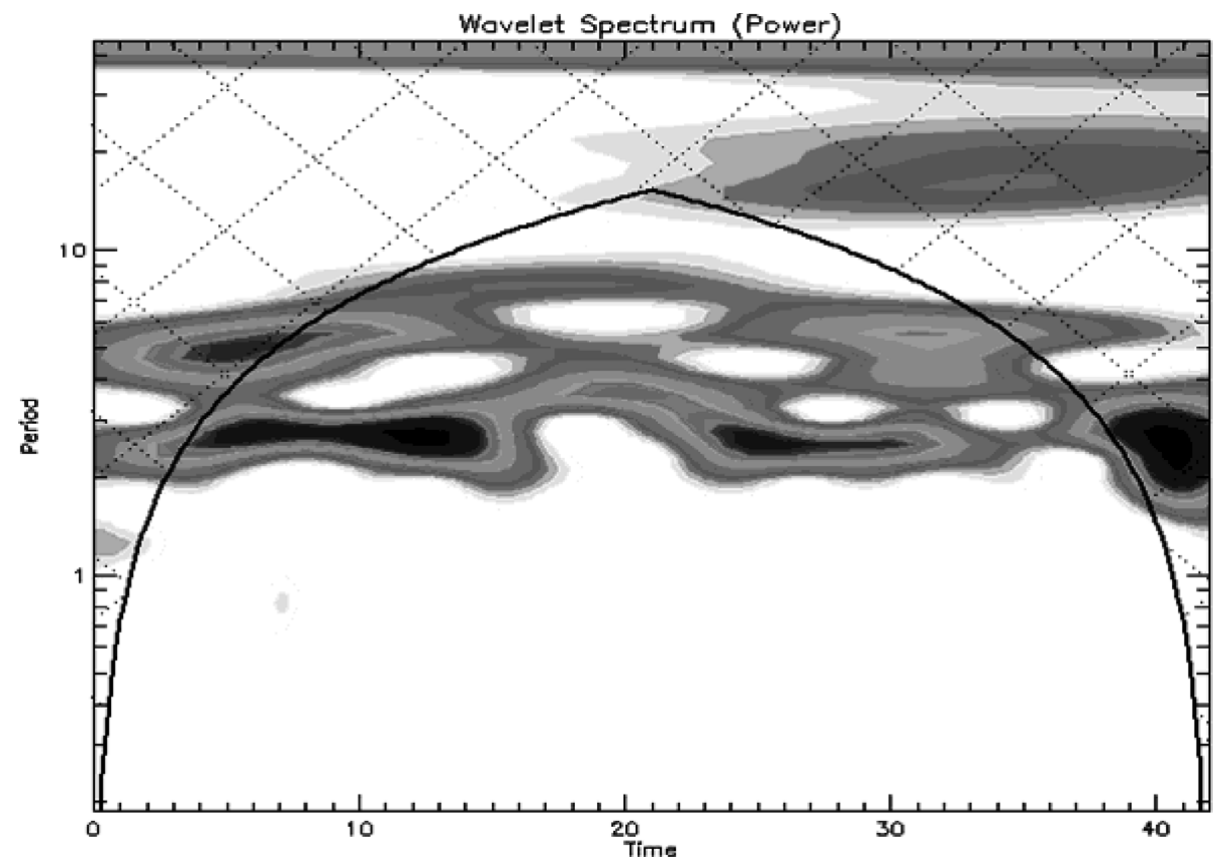

Figure 4. Wavelet spectrum of radio emission of the bright source in the active region AR10661, August 18, 2004, 01:01 - 01:43 UT.

The time profile of the longitudinal component of the chromospheric velocity is shown on Figure 1. Wavelet spectrum of time-variations of longitudinal component of the chromospheric velocity is shown on Figure 2.

Data of the Nobeyama Radioheliograph at wavelength $1.76 \mathrm{~cm}$ were used to study the radio sources placed above the same sunspots which were analyzed by optical observations. The radio maps of the whole disk were constructed for the dates of observations with the time interval of 10 seconds between the maps and $10 \mathrm{sec}$ averaging, both intensity and circular polarization maps were constructed. Having in mind that the sunspotassociated sources generated thermal gyroresonance emission at the third harmonic of the electron gyrofrequency are the most sensitive to oscillation processes, we used only the cases of the presence of such kind of sources, generated for $\lambda=1.76 \mathrm{~cm}$ in the magnetic field of $B=2000 \mathrm{G}$ at the level of the chromosphere-corona transition region (CCTR). Identification of the nature of a source is based on its high brightness temperature and strong polarization. 2D spatial resolution of the radio maps was about of $10-15$ arcsec.

The processing of radio data includes:

- imaging NoRH maps (both in total intensity $I$ and circular polarization $V$ ) with a cadence of 10 seconds (averaging 10 seconds),

- interactive extraction of a selected frame from an initial image,

- computation of the new position of the frame corrected for the solar rotation in each image according to the time of the observation,

- extraction of the frames from all images,

- computations of the time profiles of the total flux as well as the maximum brightness temperatures over each frame,

- spectral wavelet analysis of time profiles.

Variations of radio emission of the bright source in the AR 10661 (August 18, 2004, 01:01 - 01:43 UT) and wavelet spectrum are shown on Figures 3 and 4. 
Detailed comparison of wavelet spectra in radio and optics (Figures 2 and 4) shows two identical trains of the three-minute oscillations with the high level of similarity in the length and periods. At the same time the existence of their shift in time is quite evident. The radio oscillations are seen on $100 \pm 20$ seconds later than those registered by optical method. This result is in a good agreement with generally accepted idea that 3-minute oscillations demonstrate a MHD wave spreading up in the corona. Its frequency is a result of filtration in the lower underphotospheric region. In our case the velocity of these waves can be found from the observations at the level of the chromosphere and it is equal to $60 \mathrm{~km} \cdot \mathrm{sec}^{-1}$. The real velocity may be higher. So the delay of $100 \mathrm{sec}$ interpreted as the time of propagation of MHD waves from the chromosphere to corona suggests the height of the CCTR as approximately $6000 \mathrm{~km}$. This value is hardly possible to estimate by other method and height found lies in the reasonable estimations.

\section{Conclusions}

This work illustrates a possible progress in the new approach in study of quasi-periodic physical processes in the plasma structures of the active regions, especially sunspots, by comparison of parameters of the observed oscillations at different levels of the solar atmosphere. The value of the radio astronomical observations with Nobeyama Radioheliograph is determined by possibility to register the time variations of the parameters of plasma structures definitely inside the magnetic tube of sunspot at the level of the basis of the corona and by full archives of regular daily observations covering more than 15 years with practically any desirable cadence. Comparison of these observations with observations of chromospheric velocity made in $H \alpha$ line resulted in some new estimations of the 3D-dimensional structures and understanding of time evolution of the quiasy-periodic processes (MHD wave including) in magnetic tubes of a sunspots.

\section{Acknowledgement}

This work was supported by the Russian Foundation for Basic Research under grants Nos. 06-02-16838, 08-02-91860, 08-02-08744 and also by the Basic Research Program of the Presidium of the Russian Academy of Sciences No 16. V.E. Abramov-Maximov thanks IAU for financial support.

\section{References}

Bogdan, T. J. 2000, Solar Phys., 192, p. 373

Fludra, A. 2001, A\&\&A, 368, p. 639

Gelfreikh, G., Nagovitsyn, Yu. A, \& Nagovitsyna, E. Yu. 2006, PASJ, 58, p. 29

Kobanov, N. I., Kolobov, D. Y., \& Makarchik, D. V. 2006, Solar Phys., 238, p. 231

\section{Discussion}

GollA: What is your interpretation of the variation in the amplitude of the waves?

Abramov-Maximov: Probably due to mixing of different periods. 